\title{
Behavioral consequences of chronic stress and effects of antidepressant treatment on animal models of depression
}

\author{
Ritabrata Banerjee ${ }^{1}$, Somoday Hazra ${ }^{1}$, Sourav Kumar ${ }^{1}$, Anup K. Ghosh ${ }^{2}$, Amal C. Mondal ${ }^{1, ~}$ \\ ${ }^{1}$ Raja Peary Mohan College, Uttarpara, Hooghly, University of Calcutta, West Bengal-712258, India \\ ${ }^{2}$ Jadavpur University, Kolkata, West Bengal-700032, India
}

\section{Email address:}

amal_mondal@rediffmail.com(A. C. Mondal)

\section{To cite this article:}

Ritabrata Banerjee, Somoday Hazra, Sourav Kumar, Anup K. Ghosh, Amal C. Mondal. Behavioral Consequences of Chronic Stress and Effects of Antidepressant Treatment on Animal Models of Depression. American Journal of Psychiatry and Neuroscience.

Vol. 1, No. 1, 2013, pp. 5-13. doi: 10.11648/j.ajpn.20130101.12

\begin{abstract}
Major depression has a high prevalence and a high mortality. In order to understand the molecular changes underlying major depression animal models are needed. The different animal models of depression simulate the etiology and replicates symptoms, course and treatment of human depression properly. In this study, we investigated stress-induced depressogenic induction among the rats using Shuttle Box Escape Test, Open Field Test (OFT) and Elevated Plus Maze (EPM) Test. Fluoxetine hydrochloride (FLX), an antidepressant was administered chronically to determine antidepressant mediated recovery of their behavioral homeostasis. In addition, all the behavioral tests demonstrated a variety of specific symptoms like changes in locomotor activity, impaired learning ability and cognition deficit etc. From these findings, we can conclude that chronic inescapable foot-shocks at $0.8 \mathrm{~mA}$ intensity for $15 \mathrm{sec}$ duration are the most effective stressor to produce animal model of depression. After exposure to chronic foot-shocks, FLX mediated recovery strengthen our findings. In addition, the rats were screened through shuttle box escape test that mimic depressive-like behavior properly in animals. Our observation clearly corroborates well with the learned helplessness (LH) paradigm. So, the animal models of depression using electric foot-shock to induce depressive like behavior, have excellent face validity and replicate anhedonia and anergia in analogy to loss of interest and pleasure.
\end{abstract}

Keywords: Depression, Shuttle Box Escape Test, Open Field Test (OFT) and Elevated Plus Maze (EPM), Learned Helplessness (LH)

\section{Introduction}

In order to make the model of illnesses, it is helpful if the etiological basis of the disorder can be reproduced in animals. For psychiatric illnesses, this is difficult since it is a multifactorial disease. Attempts have been made using the catecholamine theory of depression to create depressive models using various means of depleting either norepinephrine or serotonin. These models have resulted in a replication of some vegetative symptoms, but are of restricted utility due to their circular nature.

In the case of depression, we do have an environmental factor that may play a role in the etiology of the illness and which has been supported by a variety of epidemiological studies in stressful life events. Thus, conditions which might lead to a usable model of depression could involve the induction of an altered behavioral stage through stress. Clues concerning the forms of stress that might be most likely to lead to depressive like behaviors can be obtained by looking at the psychological theories of depression. This study critically discusses the process of developing, validating, and working with animal models relevant to psychiatric disorders. A model is defined as any experimental preparation developed for the purpose of studying a condition in the same or different species. Typically, models are animal preparations that attempt to mimic a human condition, including human psychopathology. In developing and assessing an animal model because the intended purpose determines the criteria that the model must satisfy to establish its validity. Hence, before discussing the criteria by which the validity of an animal model may be assessed, the variety of purposes for which an animal model may be used to increase our understanding of a psychiatric disorders or its treatment that will be considered.

One can attempt to develop an animal model that mimics a psychiatric syndrome in its entirety. To do so, one must 
establish homology between the behavior of the affected animal and the syndrome being modeled. Typically, the signs and symptoms which are characteristic of the particular syndrome in humans are identified and enumerated. The ability of the experimental manipulation to induce homologous changes in the behavior of the test animal is then determined. In the early years of neuropsychopharmacology, the term animal model often denoted such an attempt to reproduce a psychiatric disorder in a laboratory animal. Furthermore, the defining symptoms of psychiatric disorders and even the diagnostic categories are being revised and re-defined continuously.

However, it is clear that each model has strengths and limitations that need to be recognized in order to use the model effectively in the investigation of psychiatric disorders. Therefore, multiple animal models are needed for each psychiatric disorder to allow investigation of the various aspects of the disease and to provide convergent validation of the research findings. Further, each model is useful for a specific purpose, for example, the identification of potential pharmacotherapy or the investigation of a specific hypothetical construct. For the purpose of antidepressant screening despair-based (Forcedswimming test and Tail suspension test), Reward-based (Sucrose preference test) and Anxiety-based (Noveltyinduced hypophagia, Open field test, Elevated plus maze test and Dark/light box test) behavioral tests are performed. On the other hand, the majority of animal models of depression are based on the exposure to various types of acute or chronic stressors, for example Learned helplessness, Chronic mild stress and Social defeat stress models are widely accepted animal models of depression.

It is widely known that stress is a risk factor for major depression in individuals with genetic vulnerability. Basic research has used animal models which imply stress to mimic such complex multi-syndrome psychiatric illnesses. They induce or mimic some of their clinical symptoms: anhedonia, sleep disturbances, increased corticosterone levels, etc. Animal models of depression are research tools used to investigate depression and action of antidepressants as a simulation to investigate the symptomatology and pathophysiology of depressive illness or used to screen novel antidepressants. An ideal animal model offers an opportunity to understand molecular, genetic, and epigenetic factors that may lead to depression. By using animal models, the underlying molecular alterations and the causal relationship between genetic or environmental alterations and depression can be examined, which would afford a better insight into pathology of depression. In addition, animal models of depression are indispensable for identifying novel therapies for depression.

It is difficult to develop an animal model that perfectly reproduces the symptoms of depression in patients. Animals lack self-consciousness, self-reflection, and consideration; moreover, hallmarks of the disorder such as depressed mood, low self-esteem or suicidality are hardly accessible in non-humans. However, depression, as other mental disorders, constitutes of endophenotypes [1] that can be reproduced independently and evaluated in animals. Endophenotype is a genetic epidemiology term which is used to parse behavioral symptoms into more stable phenotypes with a clear genetic connection. We need animal models of depression to better understand the disorders, but such models can only be developed after we understand the human disorder. Depression vulnerability genes will eventually be identified. During this interim period, one approach would be to make increasing use of animal models of particular aspects of depression, for example, cognitive or intentional impairments, or abnormalities in psychomotor activity, responses to pleasurable stimuli, and eating and sleeping behavior (Table 1). These behavioral tests have not normally been utilized in depression research and may offer new insights into the neurobiological mechanisms involved. Despite the pitfalls of available animal models of depression, these models have enabled the field to formulate several hypotheses by which depression may occur. Among all the models of depression, the Learned Helplessness (LH) model is considered to be the most preferable animal model of depression for its immense face-validity, predictive validity and reliability [2-4].

\section{Materials and Methods}

\subsection{Animals}

Male Sprague-Dawley rats were used in present experiment. At the start of the experiment rats were of the same age (approximately 2 months) weighing $224 \pm 1.5 \mathrm{gm}$. All rats were individually housed in temperature controlled $\left(22-24^{\circ} \mathrm{C}\right)$ room for at least 1 week prior to the experimentation, with ad libitum access to food and water. Rats were maintained on a $12 \mathrm{~h}$ light / dark cycle (lights on at 7am). All experimental protocols were designed to minimize the number of animals and sufferings were approved by CPCSEA and the Institutional Animal Ethics Committee (IAEC) of RPM College, Uttarpara, Hooghly, (W.B.).

The stress protocols of Lin et al., 2008, Valentine et al., 2008 and Banerjee et al., 2011a were followed [5-7]. Socially housed male rats were randomly assigned to 3 experimental groups: 1) Control group $(n=10)$ : subjected to no foot-shock throughout the experiment. They were kept in their home cage, throughout the entire behavioral paradigm. 2) Chronic stress group $(n=10)$ : received 60 foot-shocks daily for first 20 days followed by next 20 days with alternating exposure to the foot-shocks; 3) Recovery group $(n=10)$ : exposed to foot-shocks daily for 20 days and received daily injections of fluoxetine hydrochloride (FLX) for consecutive 20 days and on day 42 exposures to the foot-shock box with only the light signal to all of the rat groups (Figure 1). 


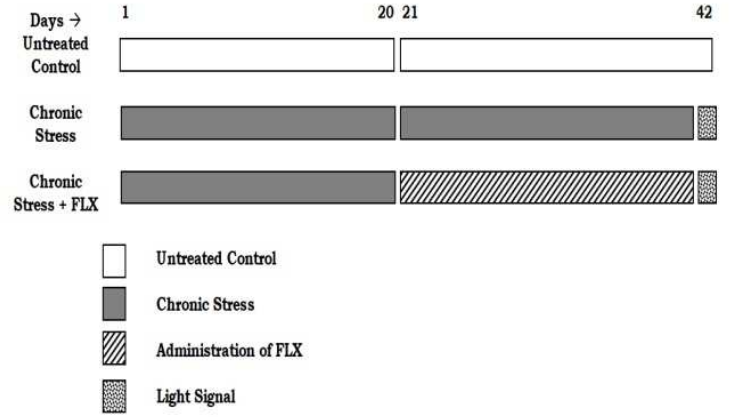

Figure 1: Schematic overview of the 42-days experimental protocol. Untreated Control: rats were subjected to no foot-shocks. Chronic Stress + FLX (Recovery): rats received foot-shocks daily for 20 days followed by a 20-day chronic antidepressant treatment instead of foot-shocks. Chronic Stress: rats received foot-shocks daily for 20 days followed by 20 days of alternating exposure to the foot-shocks. On day 42 rats of chronic and recovery groups were exposed to the foot-shock box with the light only.

\subsection{Stress Procedure}

The foot-shock chamber consists of a box containing an animal space positioned on a metallic grid floor connected to a shock generator and scrambler. Rats in stress group were placed in a box and received 60 inescapable footshocks $(0.8 \mathrm{~mA}$ intensity and $15 \mathrm{sec}$ duration with interval of 45 sec.) with randomized starting time (between 9:00h and 17:00h) and intervals during a 30 to 120 min session to make the procedure as unpredictable as possible. A light signal (15 sec) preceded each foot-shock adding a "psychological" component to the stressor. On the last day, the stress exposed rats were subjected to the light stimulus only, which was crucial as it provided a way to create a stress condition without the unwanted side effects of direct physical or painful stimuli [5]. On day 42 rats were sacrificed using isoflurane anesthesia.

\subsection{Shuttle box Escape Testing}

Shuttle box sessions were run by PC computer with custom software developed for the system (TSE Active Avoidance Systems GmbH, Bad Hamburg, Germany). At the start of each shuttle box session, animals were exposed to a 5 min habituation period in the same chamber where Inescapable shock (IS) or Escapable shock (ES) was applied. This was followed by 30 escape trials in which the gate separating the two halves of the shuttle box opened 5 sec prior to shock onset followed by randomized footshocks delivered at an intensity of $0.6 \mathrm{~mA}$ for $30 \mathrm{sec}$ duration of escape latency. The test consisted of five fixedratio 1 (FR-1) trials during which one shuttle-crossing terminated shock. FR-1 trials were used to determine the normal motor function of the rats. For escape testing, FR-1 trials were followed by 25 trials during which the rat had to cross from one side of the shuttle-box to the other, and then return, to terminate shock (fixed-ratio 2 or FR-2 trials). Shock terminated automatically if the response requirement was not met within 30 seconds of the shock onset. A mean latency for the 25 FR-2 trials of $\geq 20$ seconds are defined as learned helpless ( $\mathrm{LH})$ while mean latency of $<20$ seconds are classified as non learned helpless (NLH). Both FR-1 and FR-2 trials were presented with an average inter-trial interval of $60 \mathrm{sec}$. Crosses were automatically stored by the PC whenever a micro-switch was activated by tilting of the pivoted grid floor after crossing event. Shuttle box escape test was performed under red light conditions between 9:00h and 13:00h during the active period of the animals at least 16 hour after the last stress session and before the stress procedure of that day. The test was repeated 3 times on day1, day22 and day41. Animals of recovery group were exposed to shuttle box escape testing every third days starting after 6 days of drug treatment for a total of five shuttle box test session (day28, 31, 35, 38, 41). On testing days injections were given immediately after each shuttle box session (Figure 2).
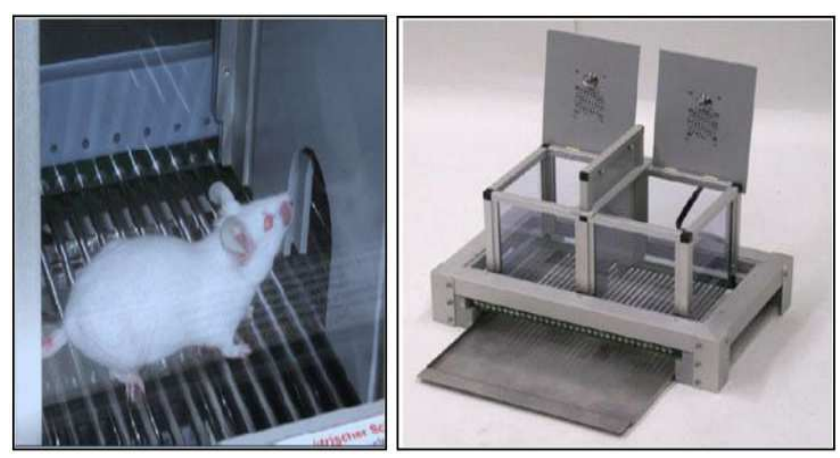

Figure 2: Shuttle box apparatus for Inescapable Foot- shocks and Escape Testing

\subsection{Chronic Antidepressant Treatment}

Fluoxetine hydrochloride, a tricyclic antidepressant drug (Sigma Aldrich, St. Louis, MI, USA) was dissolved in $0.9 \%$ physiological saline and injected intra-peritoneally (i.p) at the dose of $10 \mathrm{mg} / \mathrm{kg}$ body weight of the rats. The dosage of FLX was based on studies demonstrating a reversal of shuttle box escape deficits, after injections of FLX [2, 7-13] or exposure to chronic unpredictable shock [7-13]. Antidepressant drug was administered chronically from day 21 to day 41 once per day (Figure 3).

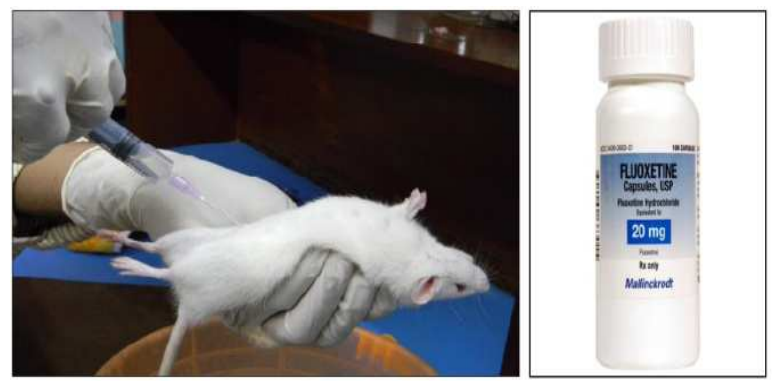

Figure 3: Fluoxetine hydrochloride (FLX) was dissolved in $0.9 \%$ physiological saline and injected intra-peritoneally (i.p) at a volume of 2 $\mathrm{ml} / \mathrm{kg}$ body weight of the rats. Drug was administered at $10 \mathrm{mg} / \mathrm{kg} /$ day on each rat. Vehicle used-0.9\% Physiological Saline. 


\subsection{Elevated Plus-Maze (EPM) Test}

The apparatus is based on that described by [14]. The maze was elevated to a height of $70 \mathrm{~cm}$ with two open $(50$ x $10 \mathrm{~cm})$ and two enclosed arms $(50 \times 10 \times 50 \mathrm{~cm})$, arranged so that the arms of the same type were opposite each other, and connected by an open central area $(10 \times 10$ $\mathrm{cm})$. Experiments were performed under dim light conditions. At the beginning of the experiment, Sprague Dawley rats were placed in the center of the maze, facing one of the enclosed arms, and observed for $4 \mathrm{~min}$. The apparatus was equipped with infrared beams and sensors capable of measuring time spent in open arms and number of open and closed-arm entries (defined as entry of all four limbs into an arm of the maze). In addition, rats were observed via video-link by an observer located in an adjacent room. This allowed the recording of a more ethologically orientated measure, namely, attempt at entry into open arms followed by avoidance responses. Data were modified using a square-root transformation, then analyzed with one-way ANOVA. Subsequent comparisons between Recovery groups, chronic stress group and untreated control group were carried out using the $t$ test. Experiments were performed $60 \mathrm{~min}$ after i.p administration of the drugs. The test was repeated 3 times on day1, day22 and day41. Animals of recovery group were exposed to Elevated Plus-Maze every third days starting after 6 days of drug treatment for a total of five test session (day28, 31, 35, 38, 41). On testing days injections were given immediately after each Elevated Plus-Maze session (Figure 4).
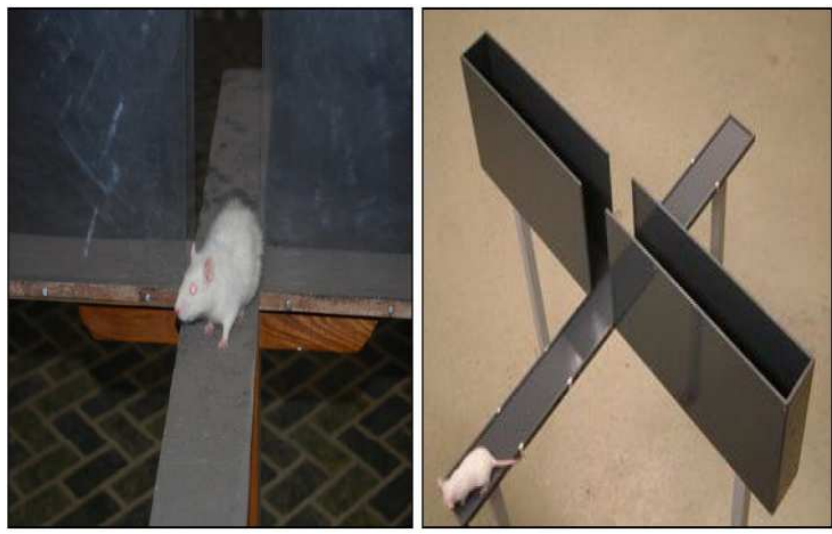

Figure 4: Elevated Plus Maze apparatus for behavioral testing

\subsection{Open Field Test (OFT)}

The open field apparatus consisted of a square wooden arena $(100 \mathrm{~cm} \times 100 \mathrm{~cm} \times 50 \mathrm{~cm})$ with a black surface covering the inside walls. The floor of the wooden arena was divided equally into 25 squares marked by black lines. In the test, a single rat was placed in the center of the arena and allowed to explore freely. The number of crossings (squares crossed with all paws) and rearings (raising of the front paws) were recorded during a test period of $5 \mathrm{~min}$. This apparatus was cleaned with a detergent and dried after occupancy by each rat. The test room was dimly illuminated (two $60 \mathrm{~W}$ lights, indirect). The computer software (EthoVision; Noldus) calculated the velocity of movement, the distance of traveling, and the time spent in the center of the open field. These parameters are thought to reflect locomotor activity and fear or anxiety, respectively (Figure 5).
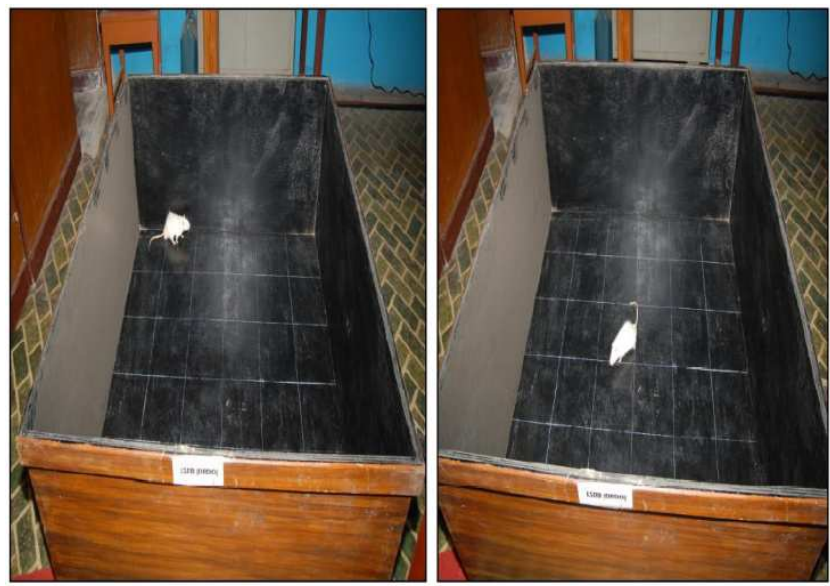

Figure 5: Open Field Test apparatus for behavioral testing

\subsection{Statistical Analyses}

The Statistical Package for the Social Science (SPSS) 15.0 was utilized for statistical analyses. All data are expressed as mean \pm standard error of $\mathrm{n}$ animals, and have been statistically analyzed with the student's $t$ - test. P values less than 0.05 were considered statistically significant. Average escape latencies of FR-1 and FR-2 trials analyzed with a repeated measured ANOVA.

\section{Results}

\subsection{Analysis of Escape Latency in Shuttle box Escape Test}

Following chronic stress exposure, the mean FR-2 escape latencies were significantly increased $(\mathrm{F} 2,165=38.37 ; \mathrm{p}<0.05)$ in the chronic stress group rats (LH) compared to respective untreated control groups and recovery rat groups which were treated with FLX, an antidepressant drug as positive control. We did not observe any significant differences in mean escape latency after FR-1 trials in any experimental paradigm (data not shown).

The mean FR-2 escape latencies were markedly differ $(t=12.98 ; \mathrm{df}=55 ; \mathrm{p}<0.001)$ in the chronic stress group compared to untreated control group.

After chronic antidepressant treatment the depressed rats of recovery group have regained their mobility and shown a significant reduction $(\mathrm{t}=5.104 ; \mathrm{df}=55 ; \mathrm{p}<0.001)$ in their escape latency compared to chronic stress group. Recovery group rats showed no significant alterations $(\mathrm{t}=3.07 ; \mathrm{df}=55$; $\mathrm{p}>0.001)$ in their escape latency compared to untreated control group (Figure 6). 


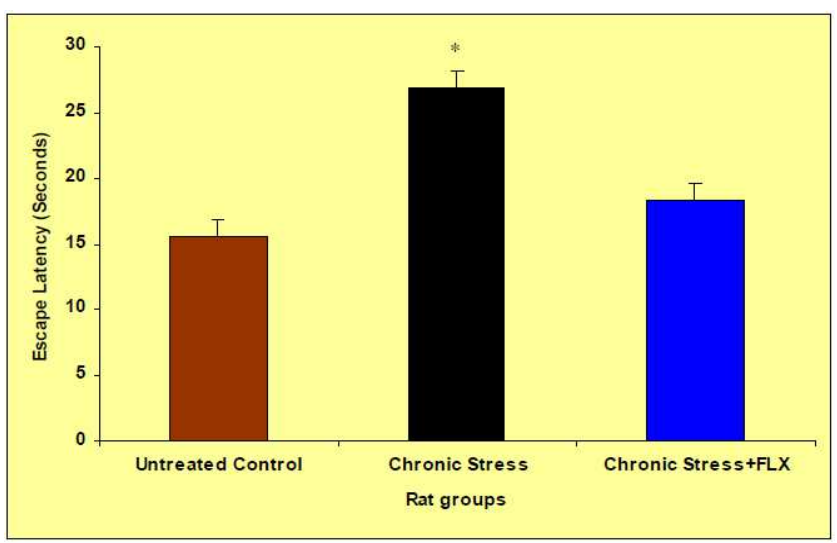

Figure 6: Effects of chronic foot-shocks and administration of FLX on FR-2 escape latencies in Shuttle box escape test in 3 experimental rat groups. The FR-2 escape latencies were significantly altered in case of chronic stress group rats $\left(F_{2,57}=188.74{ }^{*} p<0.05\right)$ than untreated controls and FLX treated individuals. Values given are the mean \pm SEMs $(n=10)$.

\subsection{Analysis of Escape Frequency in Shuttle box Escape Test}

Following chronic stress exposure, the mean FR-2 escape frequencies were significantly decreased $(\mathrm{F} 2,147=134.82 ; \mathrm{p}<0.05)$ in the chronic stress group rats (LH) compared to respective untreated control groups and recovery rat groups which were treated with FLX antidepressant drug. We did not observe any significant differences in mean escape frequency after FR-1 trials in any experimental paradigm (data not shown).

The mean FR-2 escape frequencies were markedly differ $(\mathrm{t}=18.66 ; \mathrm{df}=49 ; \mathrm{p}<0.05)$ in the chronic stress group compared to untreated control group.

After chronic antidepressant treatment the depressed rats of recovery group have regained their mobility and shown a significant enhancement $(\mathrm{t}=12.93 ; \mathrm{df}=49 ; \mathrm{p}<0.05)$ in their escape frequency compared to chronic stress group. Recovery group rats showed no significant alterations $(\mathrm{t}=1.99 ; \mathrm{df}=49 ; \mathrm{p}>0.001)$ in their escape frequency compared to untreated control group (Figure 7).

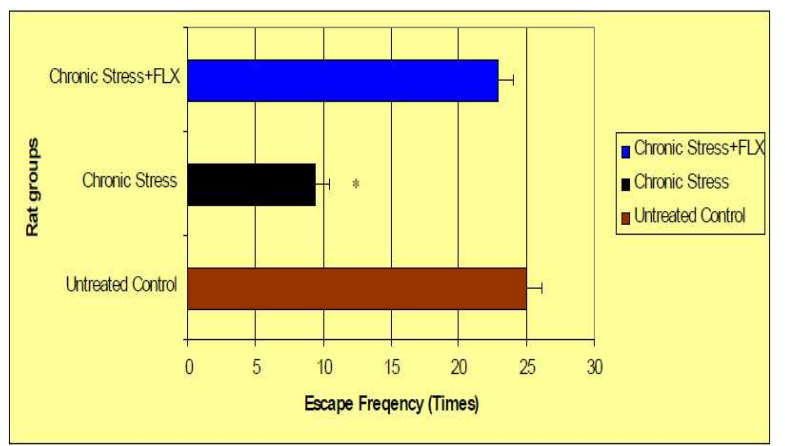

Figure 7: Effects of chronic stress and administration of FLX on FR-2 escape frequencies in Shuttle box escape test in 3 experimental rat groups. The FR-2 escape frequencies were significantly decreased in case of chronic stress group rats $\left(F_{2,147}=134.81 ;{ }^{*} p<0.05\right)$ than untreated controls and FLX treated individuals. Values given are the mean $\pm S E M s(n=10)$.

\subsection{Analysis of Open and Close Arm entry frequencies in Elevated Plus Maze Test}

Following chronic stress exposure, the open arm entry frequency was significantly decreased (F2, 57=208.51; $\mathrm{p}<0.05)$ in the chronic stress group rats $(\mathrm{LH})$ compared to respective untreated control groups and recovery rat groups treated with FLX antidepressant drug. On the other hand chronic stress group rats exhibited significant increase in their close arm entry frequency compared to other rat groups $(\mathrm{F} 2,57=13.23 ; \mathrm{p}<0.05)$.

Due to inescapable foot-shock open arm entry frequency significantly reduced among the chronic stress group rats compared to both untreated control $(\mathrm{t}=21.64 ; \mathrm{df}=19$; $\mathrm{p}<0.05)$ and recovery group $(\mathrm{t}=17.55 ; \mathrm{df}=19 ; \mathrm{p}<0.05)$ individuals. There was no significant difference in open arm entry frequencies between untreated control and recovery group rats $(\mathrm{t}=1.06 ; \mathrm{df}=19 ; \mathrm{p}>0.05)$.

Due to inescapable foot-shock close arm entry frequency significantly enhanced among the chronic stress group rats compared to both untreated control $(\mathrm{t}=7.65 ; \mathrm{df}=19 ; \mathrm{p}<0.05)$ and recovery group $(\mathrm{t}=2.86 ; \mathrm{df}=19 ; \mathrm{p}<0.05)$. There was no significant difference in close arm entry frequencies between untreated control and recovery group rats $(\mathrm{t}=1.65$; $\mathrm{df}=19 ; \mathrm{p}>0.05$ ) (Figure 8).
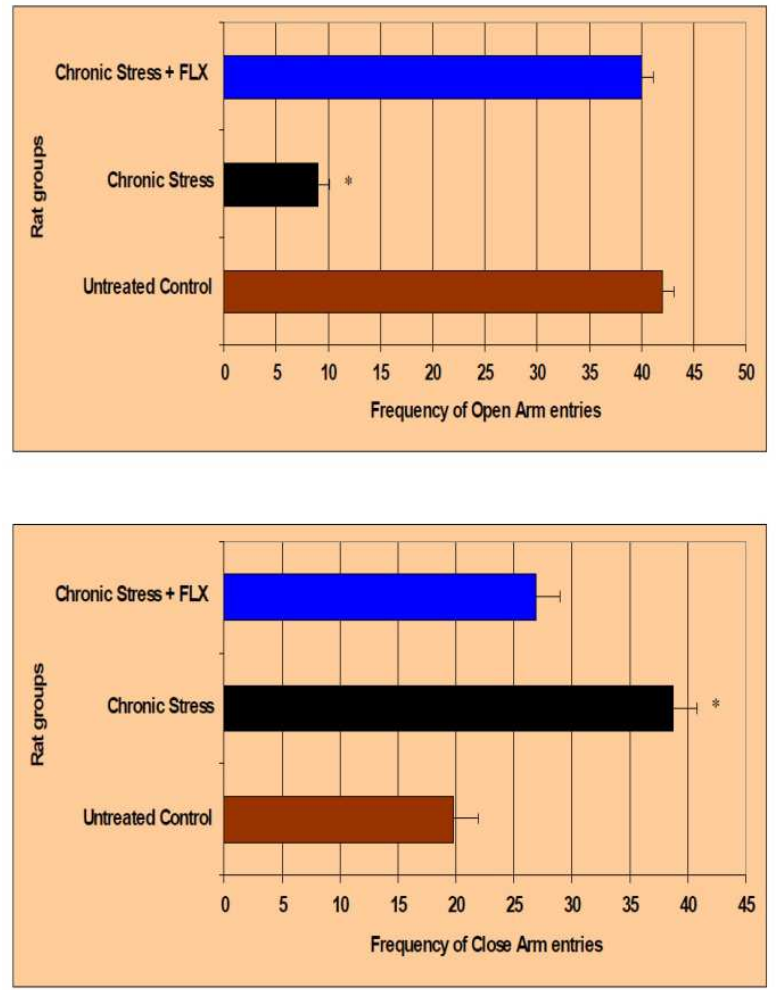

Figure 8: Effects of chronic stress and administration of FLX on open and close arm entry frequencies in Elevated Plus Maze test in 3 experimental rat groups. Open arm entry frequencies were significantly decreased in case of chronic stress group rats $\left(F_{2,57}=208.51 ; p<0.05\right)$ than untreated controls and FLX treated individuals. Close arm entry frequencies were significantly increased in case of chronic stress group rats $\left(F_{2,57}=13.23\right.$; $p<0.05)$ than untreated controls and FLX treated individuals. Values given are the mean \pm SEMs $(n=10)$. 


\subsection{Analysis of Time Spent in Open and Close Arm in Elevated Plus Maze Test}

Following chronic stress exposure, the duration of time spent in open arm was significantly decreased (F2, $57=71.13 ; \mathrm{p}<0.05)$ in the chronic stress group rats $(\mathrm{LH})$ compared to respective untreated control groups and recovery rat groups which were treated with FLX antidepressant drug. On the other hand, chronic stress group rats preferred to spent their time more in the close arms during the test period. The entire time spent in close arm is significantly higher than the other rat groups (F2, 57=48.68; $\mathrm{p}<0.05)$.

Due to inescapable foot-shock open arm time spent significantly reduced among the chronic stress group rats compared to both untreated control $(\mathrm{t}=16.91 ; \mathrm{df}=19$; $\mathrm{p}<0.05)$ and recovery group $(\mathrm{t}=9.08 ; \mathrm{df}=19 ; \mathrm{p}<0.05)$ individuals. There was no significant difference in open arm time spent between untreated control and recovery group rats $(\mathrm{t}=1.53 ; \mathrm{df}=19 ; \mathrm{p}>0.05)$ (Figure 9).

Due to inescapable foot-shock close arm entry frequency significantly enhanced among the chronic stress group rats compared to both untreated control $(\mathrm{t}=8.31 ; \mathrm{df}=19 ; \mathrm{p}<0.05)$ and recovery group $(\mathrm{t}=9.75 ; \mathrm{df}=19 ; \mathrm{p}<0.05)$ individuals. There was no significant difference in close arm entry frequencies between untreated control and recovery group rats $(\mathrm{t}=0.31 ; \mathrm{df}=19 ; \mathrm{p}>0.05)$ (Figure 9).

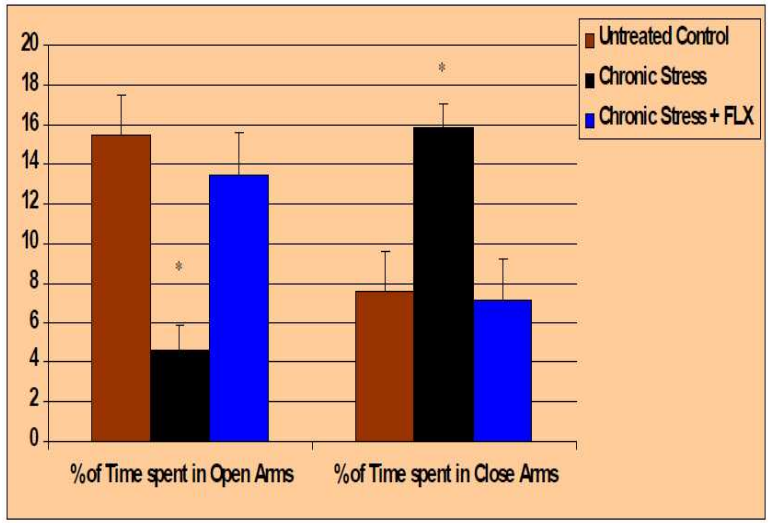

Figure 9: Effects of chronic stress and administration of FLX on open \& close arm time spent in Elevated Plus Maze test in 3 experimental rat groups. Close arm time spent was significantly increased in case of chronic stress group rats $\left(F_{2,57}=48.68 ; p<0.05\right)$ than untreated controls and FLX treated individuals whereas Open arm time spent was significantly decreased in case of chronic stress group rats $\left(F_{2,57}=71.13\right.$; $p<0.05)$ than untreated controls and FLX treated individuals. Values given are the mean \pm SEMs $(n=10)$.

\subsection{Analysis of Number of aborted Attempts in Elevated Plus Maze Test}

Following chronic stress exposure, the frequency of aborted attempt was significantly increased (F2, 57=82.00; $\mathrm{p}<0.05)$ in the chronic stress group rats $(\mathrm{LH})$ compared to respective untreated control groups and recovery rat groups which were treated with FLX antidepressant drug.

Due to inescapable foot-shock frequency of aborted attempts significantly increased among the chronic stress group rats compared to both untreated control $(\mathrm{t}=11.22$; $\mathrm{df}=19 ; \mathrm{p}<0.05)$ and recovery group $(\mathrm{t}=9.04 ; \mathrm{df}=19 ; \mathrm{p}<0.05)$ individuals.

Due to chronic administration of FLX frequency of aborted attempts significantly reduced among the recovery group rats compared to chronic stress group individuals. There was no significant difference in aborted attempts frequency between untreated control and recovery group rats $(\mathrm{t}=0.41 ; \mathrm{df}=19 ; \mathrm{p}>0.05)$ (Figure 10$)$.

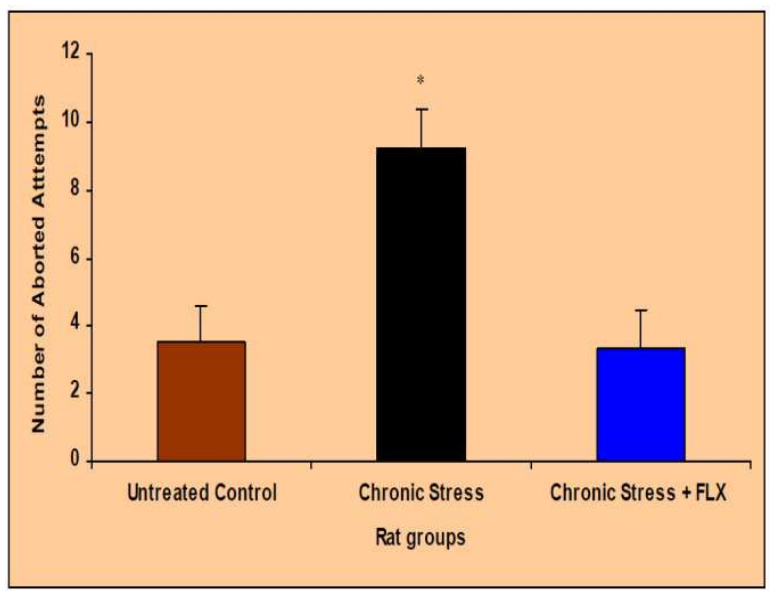

Figure 10: Effects of chronic stress and administration of FLX on the number of aborted attempts in Elevated Plus Maze test in 3 experimental rat groups. The aborted attempts frequency was significantly enhanced in case of chronic stress group rats $\left(F_{2,57}=82.00 ;{ }^{*} p<0.05\right)$ than untreated controls and FLX treated individuals. Values given are the mean \pm SEMs (n $=10)$.

\subsection{Analysis of Number of Crossings and Rearings in Open Field Test}

This study showed the effect of chronic stress and FLX treatment on locomotor activity among the rats. Locomotor activity was assessed by the number of crossings and rearings in the open field test. Before inescapable footshock exposure there was no significant difference in the number of crossings and rearings among all rats (data not shown). Whereas the significant reduction in the number of crossings $(\mathrm{F} 2, \quad 57=62.56 ; \mathrm{p}<0.05)$ and rearings $(\mathrm{F} 2$, $57=44.28 ; \mathrm{p}<0.05)$ were observed after 3 weeks exposure to inescapable chronic foot-shocks among 3 experimental rat groups.

\subsubsection{Effect of Inescapable Foot-shocks and Chronic Anti-depressant treatment on the number of Crossings}

Due to inescapable foot-shock frequency of crossing significantly decreased among the chronic stress group rats compared to both untreated control $(\mathrm{t}=19.47 ; \mathrm{df}=19$; $\mathrm{p}<0.05)$. and recovery group $(\mathrm{t}=6.68 ; \mathrm{df}=19 ; \mathrm{p}<0.05)$ individuals. There was no significant alteration $(\mathrm{t}=1.91$; $\mathrm{df}=19 ; \mathrm{p}>0.05)$ in crossing frequency was observed between untreated control and recovery group rats (Figure 11). 


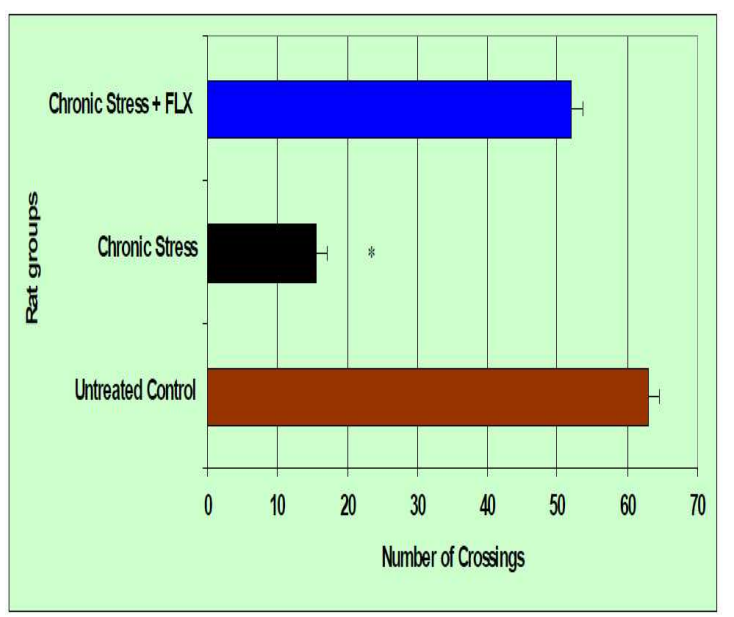

Figure 11: Effects of chronic stress and administration of FLX on Number of Crossings in Open Field Test in 3 experimental rat groups. Crossing frequencies were significantly decreased in case of chronic stress group rats $\left(F_{2,57}=62.56 ; p<0.05\right)$ than untreated controls and FLX treated individuals. Values given are the mean \pm SEMs $(n=10)$.

\subsubsection{Effects of Inescapable Foot-shocks and Chronic Anti-depressant treatment on the number of Rearings}

Due to inescapable foot-shock frequency of rearing significantly decreased among the chronic stress group rats compared to both untreated control $(t=9.26 ; \mathrm{df}=19 ; \mathrm{p}<0.05)$ and recovery group $(\mathrm{t}=5.99 ; \mathrm{df}=19 ; \mathrm{p}<0.05)$ individuals. There was no significant alteration $(t=1.75 ; d f=19 ; p>0.05)$ in rearing frequency was observed between untreated control and recovery group rats (Figure 12).

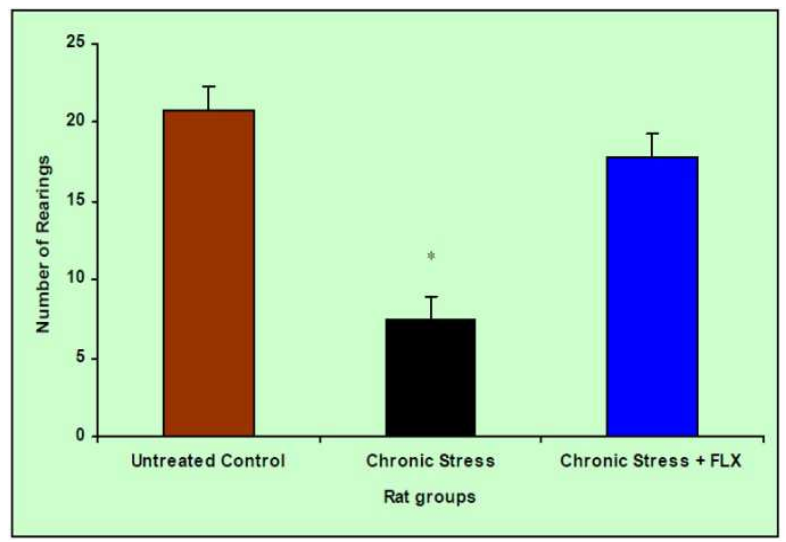

Figure 12: Effects of chronic stress and administration of FLX on number of rearings in Open Field Test in 3 experimental rat groups. Crossing frequencies were significantly decreased in case of chronic stress group rats $\left(F_{2,57}=44.28 ; p<0.05\right)$ than untreated controls and FLX treated individuals. Values given are the mean $\pm \operatorname{SEMs}(n=10)$.

\section{Discussion}

These studies are solely based on chronic stress induced depressive behaviors and the effects of chronic antidepressant drug to recover the state of anxiety. Among the despair-based test due to having poor face and construct validities and sensitivity to only acute antidepressant treatment forced-swimming test and tail suspension test cannot be considered in this experiment. On the other hand, the anxiety-based tests can be used as an index of anxiolytic activity but it is difficult to discriminate decreased anxiety-related avoidance from increased novelty-seeking in these tests.

Results obtained from EPM test clearly indicates that an increase tendency of rodents to enter in the open-arm and their time spent in that arm is an index of anti-anxiety behavior of rodents. Similarly, the observations of OFT demonstrates the tendency of rodents to avoid brightly illuminated areas, and this avoidance is confused as a symptom of anxiety. Obtained results from these tests are clearly corroborated with the helpless behavior in $\mathrm{LH}$ model, which was evaluated by analyzing the performance in an active escape test, such as the latency to press a lever or cross a door. Considering all the results of these three tests, it may be concluded that 60 foot-shocks daily for first 20 days followed by next 20 days with alternating exposure to the foot-shocks exposure is solely responsible to implicit such depressive-like state among the rodents.

An advantage of LH model is that the cognitive and other behavioral outcomes seem to be correlated, thus helping to understand the depressive symptomatology in humans. Besides, this model can also be generally used to measure the escape performance of mice with different mutations, in which target genes of depression may affect the vulnerability to develop a depressive-like state. These excellent face and predictive validities make $\mathrm{LH}$ an interesting model to explore the pathophysiology of depression. Learned helplessness, as a technical term in animal psychology and related with human psychology, means a condition of a human being or an animal in which it has learned to behave helplessly, even when the opportunity is restored for it to help itself by avoiding an unpleasant or harmful circumstance to which it has been subjected.

The most widely accepted theories are cognitive theories of depression that suggest that depression results through a misinterpretation of cognitive information which is evaluated as being much more negative than is actually the case. This leads to a feeling of helplessness and a sense that one has lost control. This suggests that using stress to induce a feeling of loss of control might result in a behavioral state analogous to depression. The best animal model of depression simulates the etiology and replicates symptoms, course and treatment of human depression. The most utilized and validated model of depression involving the application of uncontrollable stress, which might be expected to lead to a depressive behavioral state, is the Learned helplessness model of depression [15-18].

Considering immense validity and reliability of depressive-like states in Learned Helplessness (LH) models of depression, this animal model of depression may be considered for further analyses of molecular mechanism of depression. The rationality of the chronic stress induced LH model is already proved and vigorously accepted to 
study depressive-like states in animals.

Helpless animals have loss of weight, agitation, decreased locomotor activity, sleep disturbances, decreased libido, reduced learning in some tests but not in spatial learning tests, and alterations in the HPA axis, with elevated corticosterone. Anhedonia has been postulated as a central symptom of depression, which can be monitored in animals using sucrose preference tests [19-20]. Though there may be some debate as to how central preference of sweet solutions is as a measure of the depressive state, it has been measured in helpless animals and they have been found to consume less sucrose or saccharin than control animals $[2-4,21]$. Furthermore, anhedonia has been demonstrated by increased thresholds to intracranial selfstimulation after uncontrollable foot-shock [22]. Thus, all measures that reflect depressive symptoms or physiological changes associated with depression are found in learned helplessness model. This model appears to mirror findings seen in depressive illness in man in most aspects that can be examined in animals.

The model also has had extensive tests of predictive validity with all classes of antidepressants, electroconvulsive therapies and even cognitive training. The model has been found useful in testing compounds for antidepressant activity [23]. A variety of tricyclic antidepressants have been tested and found to be effective in reversing helplessness. These include imipramine, a combined norpinephrine and 5HT reuptake inhibitor, desipramine acting predominately on norepinephrine transport sites and amytriptyline, acting more on 5HT sites. The specific 5HT reuptake inhibitors have also been examined and found to reverse helplessness, including fluoxetine.

\section{Conclusion}

The present study shows that the Learned Helplessness (LH) model becomes a more valid pharmacological model to determine behavioral, physiological and neurochemical aspects of depression. The LH model has the advantage of screening antidepressant properties of drugs that is needed to recover from depressive-like state than EPM and OFT. Our observation clearly corroborates with the previous findings of the scientists [23-33], and forecasts the rationality of inescapable foot-shocks induced LH model of depression.

\section{Acknowledgements}

This research work was financially supported by grants from SERB (DST) [SR/SO/HS-57/2008], Govt. of India and LSRBDRDO [DLS/81/48222/LSRB-246/EPB/2012], and Special thanks to the authorities of RPM College, Uttarpara, Hooghly, (W.B.) and Jadavpur University, Kolkata. This work was performed as a doctoral thesis for R. Banerjee.

\section{References}

[1] Hasler G., Drevets WC., Manji HK., Charney DS. Discovering endophenotypes for major depression. Neuopsychopharmacol. 2004; 29:1765-1781.

[2] Gambarana C., Scheggi S., Tagliamonte A., Tolu P., De Montis MG. Animal models for the study of antidepressant activity. Brain Res Protoc 2001;7:11-20.

[3] Minor TR., Dess NK., Ben David E., Chang WC. Individual differences in vulnerability to inescapable shock in rats. J Exp Psychol Anim Behav Proc 1994; 20:402-12.

[4] Vollmayr B., Bachteler D., Vengeliene V., Gass P., Spanagel R., Henn F. Rats with congenital learned helplessness respond less to sucrose but show no deficits in activity or learning. Behav Br Res. 2004; 150: 217-221.

[5] Lin Y., Westenbroek C., Bakker P., Termeer J., Liu A., Li X. and Ter Horst GJ. Effects of long-term stress and recovery on the prefrontal cortex and dentate gyrus in male and female rats. Cereb cortex. 2008; 18: 2762-2774.

[6] Valentine G., Dow A., Banasr M., Pittman B., Duman R. Differential effects of chronic antidepressant treatment on shuttle box escape deficits induced by uncontrollable stress. Psychopharmacol. 2008; 200: 585-596.

[7] Banerjee R., Ghosh AK., Ghosh B., Mondal AC. FemaleSpecific Depression Induction in Learned-Helplessness Model of Rats. Int J Bioeng Sci and Tech 2011a; 2(3): 5159.

[8] Banerjee R., Ghosh AK., Ghosh B., Mondal AC. Stress: The Negative modulator of NGF. Res Rev: A J Life Sci. 2011b; $1(2): 1-9$.

[9] Banerjee R., Das M., Mondal AC., Ghosh B., Ghosh AK. Influences of chronic stress and antidepressant treatment on the hippocampal concentrations of macromolecule BDNF (Brain-derived neurotrophic factor) in a rat model of Learned helplessness Asian J Microbio, Biotech Env Sci. 2011c 13(4): 625-632.

[10] Banerjee R., Ghosh AK., Mondal AC. Effects of chronic stress and antidepressant treatment on behavioral, physiological and neurochemical aspects in male and female rats. Al Ameen J Med Sci. 2012a; 5(2):165-176.

[11] Banerjee R, Ghosh AK, Ghosh B, Batabyal S, Mondal AC. Effects of chronic mild stress on brain derived neurotrophic and nerve growth factors in the rat hippocampus. Neurosci Res Lett.. 2012b; 3(1): 29-34.

[12] Gambarana C., Scheggi S., Tagliamonte A., Tolu P., De Montis MG. Animal models for the study of antidepressant activity. Brain Res Protoc. 2001; 7: 11-20.

[13] Chen H., Pandey GN., Dwivedi Y. Hippocampal cell proliferation regulation by repeated stress and antidepressants. Neuroreport. 2006; 17: 863-867.

[14] Banerjee R., Ghosh AK., Mondal AC. Chronic administration of Fluoxetine ameliorates depression: Enhanced BDNF and its receptor TrkB expressions with down stream signalling cascades ERK1/2 and Akt pathways. J Pharm Biomed Sci. 2013; 5(2):165-176. 
[15] Pellow S., Chopin P., File SE., Briley M. Validation of open:closed arm entries in an elevated plus-maze as a measure of anxiety in the rat. J Neurosci Methods. 1985; $14: 149-167$.

[16] Drugan RC., Basile AS., Ha JH., Healy D., Ferland RJ. Analysis of the importance of controllable versus uncontrollable stress on subsequent behavioral and physiological functioning. Brain Res Brain Res Protoc. 1997; 2(1):69-74.

[17] Grahn RE., Watkins LR., Maier SF. (2000) Impaired escape performance and enhanced conditioned fear in rats following exposure to an uncontrollable stressor are mediated by glutamate and nitric oxide in the dorsal raphe nucleus. Behav Brain Res. 2000; 112(1-2): 33-41.

[18] Durgam RC. Rodent models of depression: learnedhelplessness using a triadic design in rats. Curr Protoc Neurosci. 2001; Chapter 8:Unit 8.10B.

[19] Chourbaji S., Zacher C., Sanchis-Segura C., Dormann C., Vollmayr B., Gass P. Learned helplessness: validity and reliability of depressive-like states in mice. Brain Res Brain Res Protoc. 2005; 16(1-3): 70-78.

[20] Willner P. Animal models of depression: an overview. Pharmacol Ther. 1990; 45:425-55.

[21] Willner P. Animal models of depression: validity and applications. Adv Biochem Psychopharmacol. 1995; 49: $19-41$.

[22] Vollmayr B., Henn FA. Learned helplessness in the rat: improvements in validity and reliability. Brain Res Brain Res Protoc. 2001; 8(1): 1-7.

[23] Zacharko RM., Anisman H. Stressor-induced anhedonia in the mesocorticolimbic system. Neurosci Biobehav Rev. 1991; 15: 391-405.
[24] Sherman AD., Sacquitne JL., Petty F. Specificity of the learned helplessness model of depression. Pharmacol Biochem Behav. 1982; 16: 449-454.

[25] Greenberg L, Edwards E, Henn FA. (1989) Dexamethasone suppression test in helpless rats. Biol Psychiatry 26:530532.

[26] Edwards E., Harkins K., Wright G., Henn F. Effects of bilateral adrenalectomy on the induction of learned helplessness behavior. Neuropsychopharmacol. 1990; 3: 109-114.

[27] Duman RS., Malberg J., Nakagawa S., D’Sa C. Neuronal plasticity and survival in mood disorders. Biol Psychiatry 2000; 48: 732-739.

[28] Manji HK.., Drevets WC.., Charney DS. The cellular neurobiology of depression. Nat Med 2001; 7: 541-547.

[29] Coyle JT., Duman RS. Finding the intracellular signaling pathways affected by mood disorder treatments. Neuron 2003; 38: 157-160.

[30] Shirayama Y., Chen AC., Nakagawa S., Russell DS., Duman RS. Brainderived neurotrophic factor produces antidepressant effects in behavioral models of depression. $\mathrm{J}$ Neurosci 2002; 22: 3251-3261.

[31] Siuciak JA., Lewis DR., Wiegand SJ., Lindsay RM. Antidepressant-like effect of brain-derived neurotrophic factor (BDNF). Pharmacol Biochem Behav. 1997; 56: 131137.

[32] Fuchs E., Gould E. Mini-review: in vivo neurogenesis in the adult brain: regulation and functional implications. Eur $\mathrm{J}$ Neurosci. 2000; 12: 2211-2214.

[33] Gould E., Tanapat P., Rydel T., Hastings N. Regulation of hippocampal neurogenesis in adulthood. Biol Psychiatry. 2000; 48: 715-720. 